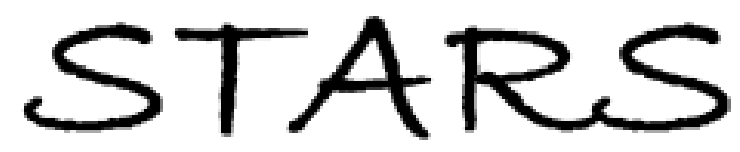

University of Central Florida

STARS

Faculty Bibliography 2010s

Faculty Bibliography

$1-1-2013$

\title{
Phase-unlocked Hong-Ou-Mandel interferometry
}

Ayman F. Abouraddy

University of Central Florida

Timothy M. Yarnall

Giovanni Di Giuseppe

University of Central Florida

Find similar works at: https://stars.library.ucf.edu/facultybib2010

University of Central Florida Libraries http://library.ucf.edu

This Article is brought to you for free and open access by the Faculty Bibliography at STARS. It has been accepted for inclusion in Faculty Bibliography 2010 s by an authorized administrator of STARS. For more information, please contactSTARS@ucf.edu.

\section{Recommended Citation}

Abouraddy, Ayman F.; Yarnall, Timothy M.; and Giuseppe, Giovanni Di, "Phase-unlocked Hong-Ou-Mandel interferometry" (2013). Faculty Bibliography 2010s. 3582.

https://stars.library.ucf.edu/facultybib2010/3582

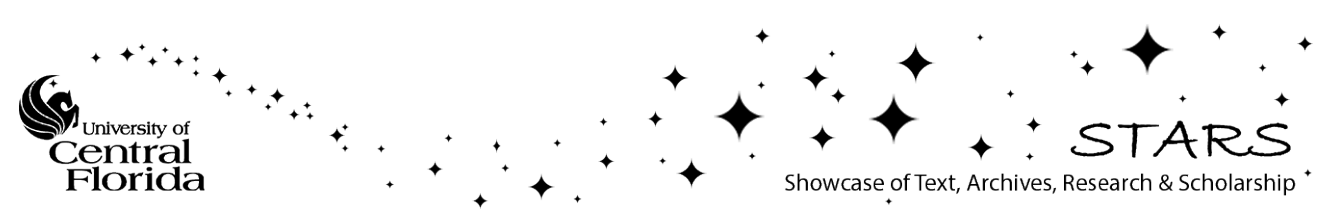




\title{
Phase-unlocked Hong-Ou-Mandel interferometry
}

\author{
Ayman F. Abouraddy, ${ }^{1, *}$ Timothy M. Yarnall, ${ }^{2, \dagger}$ and Giovanni Di Giuseppe ${ }^{1,3}$ \\ ${ }^{1}$ CREOL, College of Optics \& Photonics, University of Central Florida, Orlando, Florida 32816, USA \\ ${ }^{2} 19$ Chestnut Circle, Merrimack, New Hampshire 03054, USA \\ ${ }^{3}$ School of Science and Technology, Physics Division, University of Camerino, 62032 Camerino, Italy
}

(Received 23 August 2012; published 11 June 2013)

\begin{abstract}
There is a fundamental dimensional mismatch between the Hong-Ou-Mandel (HOM) interferometer and two-photon (2P) states: while the latter are represented using two temporal (or spectral) dimensions, the HOM interferometer allows access to only one temporal dimension. We introduce a linear $2 \mathrm{P}$ interferometer containing two independent delays spanning the $2 \mathrm{P}$ state. By "unlocking" the fixed phase relationship between the interfering 2P probability amplitudes in a HOM interferometer, one of these probability amplitudes now serves as a delay-free $2 \mathrm{P}$ reference against which the other beats, thereby resolving ambiguities in $2 \mathrm{P}$ state identification typical of HOM interferometry and extending its utility to a large family of $2 \mathrm{P}$ states.
\end{abstract}

DOI: 10.1103/PhysRevA.87.062106

PACS number(s): 42.50.St, 03.67.Bg, 42.50.Dv

The Hong-Ou-Mandel interferometer (HOM-I), conceived 25 years ago [1], is now a centerpiece of quantum optics. In its original conception, a relative delay is swept between two photons brought together at a beam splitter (BS) and coincidence measurements typically reveal a "dip," indicating that the two photons emerge together from either BS port [2]. The HOM-I has played an instrumental role in studying multiphoton interference [3] and testing the foundations of quantum mechanics [4] and in applications of photonic quantum information processing that require temporal overlap of photon wave packets [5].

The HOM-I has a salient feature in common with classical linear interferometers: a one-dimensional (1D) interferogram is produced by sweeping a delay. Since two-photon (2P) states have a two-dimensional (2D) frequency spectrum with one frequency characterizing each photon, there is a fundamental dimensional mismatch between $2 \mathrm{P}$ states and the HOM-I. The HOM-I limits access to a 1D slice through the 2P state, leading to ambiguities in $2 \mathrm{P}$ state identification. Moreover, some 2P states yield a featureless HOM interferogram, such as frequency-correlated states [6]. The realization that the HOM-I does not provide adequate characterization of an arbitrary $2 \mathrm{P}$ state, coupled with the introduction of various engineered $2 \mathrm{P}$ states [7-10] using the process of optical spontaneous parametric downconversion (SPDC) from a nonlinear crystal (NLC) [11], has led to alternative efforts relying on nonlinear phenomena such as sum-frequency generation [12] and femtosecond upconversion [13] of the photon pair. Additionally the joint $2 \mathrm{P}$ spectrum has been directly measured by spectral coincidence measurements [14] and extracted from 2P interference in uncoupled one-photon interferometers $[15,16]$. There remains, nevertheless, important motivation for linear time-domain measurements in applications requiring photon synchronization [5], clock synchronization [17], and metrology [18], besides the improved signal-to-noise-ratio and precision enabled by temporal interferometry.

\footnotetext{
*raddy@creol.ucf.edu

${ }^{\dagger}$ Currently with the Massachusetts Institute of Technology, Lincoln Laboratory, Lexington, MA 02420, USA.
}

In this paper we describe a linear dual-delay $2 \mathrm{P}$ interferometer that spans both temporal dimensions of a $2 \mathrm{P}$ state and differs from the HOM-I in two fundamental ways. First, sweeping two delays in the proposed interferometer produces a $2 \mathrm{D}$ interferogram that reduces to the $1 \mathrm{D} \mathrm{HOM}$ interferogram when these two delays are swept in opposition. Sweeping the two delays in unison, on the other hand, reveals an "orthogonal" temporal interferogram that has been inaccessible to the traditional HOM-I. While the interfering $2 \mathrm{P}$ probability amplitudes in the HOM-I are locked in antiphase, the corresponding probability amplitudes in our interferometer are independent. We thus refer to this interferometer as a phase-unlocked HOM-I (PhuL HOM-I). Such a scheme allows synchronizing the two photons regardless of their state. Secondly, 2P interference occurs in the PhuL HOM-I without the photons meeting at BSs or even sharing a common path. The notion that the two photons "stick" to each other when meeting at the BS in the HOM-I was dispelled in Ref. [19] through manipulation of multiple delays in a HOM-I. We demonstrate here that the interfering HOM probability amplitudes need not share any common paths altogether.

Consider the following 2P state in frequency space:

$$
|\Psi\rangle=\iint d \omega_{1} d \omega_{2} \Phi\left(\omega_{1}, \omega_{2}\right)\left|1_{\omega_{1}}, 1_{\omega_{2}}\right\rangle,
$$

where $\iint d \omega_{1} d \omega_{2}\left|\Phi\left(\omega_{1}, \omega_{2}\right)\right|^{2}=1$. The time-averaged coincidence rate [20] registered by detectors $\mathrm{D}_{1}$ and $\mathrm{D}_{2}$ in an HOM-I [Fig. 1(a)] is $G_{12}^{(2)}(\tau)=1-\Lambda(\tau)$, where

$$
\Lambda(\tau)=\iint d \omega_{1} d \omega_{2} \Phi\left(\omega_{1}, \omega_{2}\right) \Phi^{*}\left(\omega_{2}, \omega_{1}\right) e^{-i\left(\omega_{1}-\omega_{2}\right) \tau} .
$$

The interferogram visibility $V=\Lambda(0)$ indicates the indistinguishability of the two photons and is maximized when the state is symmetric under photon exchange, $\Phi\left(\omega_{1}, \omega_{2}\right)=$ $\Phi\left(\omega_{2}, \omega_{1}\right)$. This outcome stems from the destructive interference of two $2 \mathrm{P}$ probability amplitudes: both photons are reflected by the BS to $\mathrm{D}_{1}$ and $\mathrm{D}_{2}$ [Fig. 1(b)] or transmitted through the BS [Fig. 1(c)], resulting in the absence of coincidence counts. The HOM interference term [Eq. (2)] is the 2D Fourier transform, $\left(\omega_{1}, \omega_{2}\right) \rightarrow\left(\tau_{1}, \tau_{2}\right)$, of the symmetrized state function $\Phi\left(\omega_{1}, \omega_{2}\right) \Phi^{*}\left(\omega_{2}, \omega_{1}\right)$ evaluated only along the 


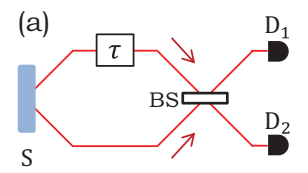

(b)
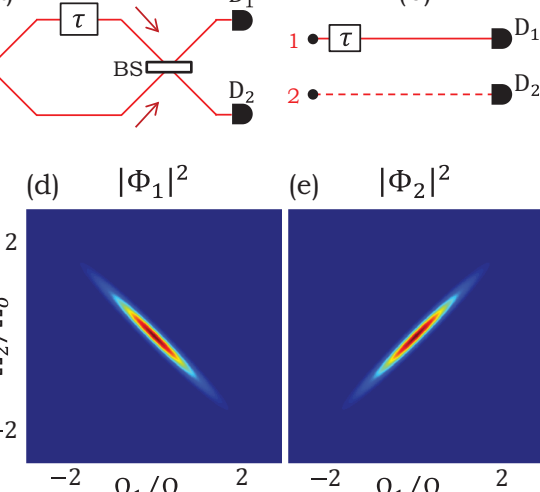

(e) $\left|\Phi_{2}\right|^{2}$

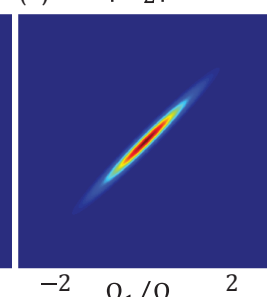

(h) (c)

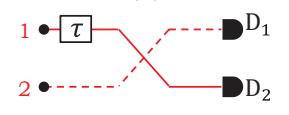

(f) $\left|\Phi_{3}\right|^{2}$

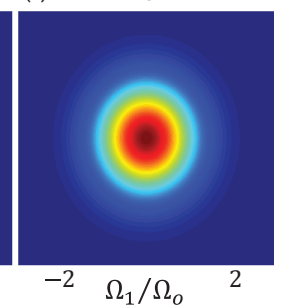

(i)

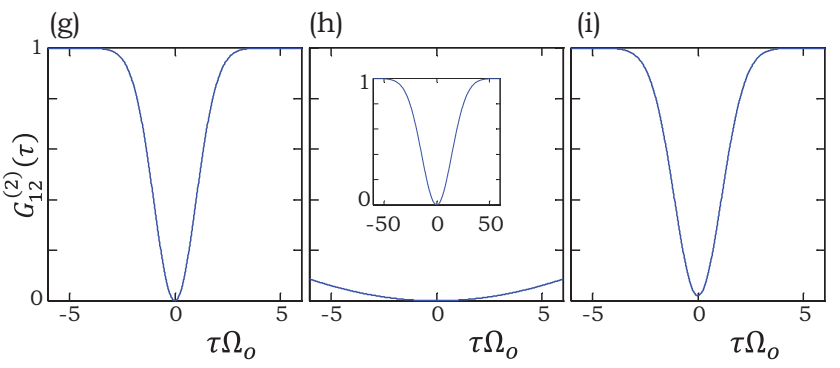

FIG. 1. (Color online) (a) Schematic of a HOM-I; $\mathrm{S}$ is a $2 \mathrm{P}$ source, BS: beam splitter, $\tau$ : optical delay. (b), (c) The interfering 2P probability amplitudes that produce the HOM "dip." (d)-(f) Frequency-domain $2 \mathrm{P}$ state functions $\left|\Phi\left(\Omega_{1}, \Omega_{2}\right)\right|^{2}$, and (g)-(i) the corresponding HOM interferograms $G_{12}^{(2)}(\tau)$. (d), (g) A 2P entangled frequency-anticorrelated state $\Phi_{1}\left(\theta=-\frac{\pi}{4}\right.$ and $\left.\xi=0.1\right)$; (e), (h) a 2P entangled frequency-correlated state $\Phi_{2}\left(\theta=\frac{\pi}{4}\right.$ and $\left.\xi=0.1\right)$; and (f), (i) a $2 \mathrm{P}$ separable state $\Phi_{3}(\theta=0$ and $\xi=2)$. The inset in (h) shows $G_{12}^{(2)}(\tau)$ for extended $\tau$.

diagonal $\tau=\tau_{1}=-\tau_{2}$. Consequently the HOM-I does not distinguish between $2 \mathrm{P}$ states that have the same values along this diagonal but are otherwise different.

To elucidate this fact, we consider a model state that captures the physics of a wide range of $2 \mathrm{P}$ states,

$$
\Phi\left(\Omega_{1}, \Omega_{2}\right)=\eta e^{-\frac{\left(\cos \theta \Omega_{1}-\sin \theta \Omega_{2}\right)^{2}}{2 \Omega_{\mathrm{c}}^{2}}} e^{-\frac{\Omega_{1}^{2}+\Omega_{2}^{2}}{2 \Omega_{\mathrm{o}}^{2}}} ;
$$

where we have shifted the frequencies, $\omega_{j}=\omega_{0}+\Omega_{j} \rightarrow \Omega_{j}$, $j=1,2, \omega_{0}$ and $\Omega_{0}$ are the central optical frequency and bandwidth of each photon, respectively, $\Omega_{\mathrm{c}}$ is the correlation uncertainty bandwidth along a line at an angle $\theta$ with the $\Omega_{1}$ axis, and $\eta$ is a normalization constant, $\eta^{4}=\frac{1}{\Omega_{0}^{2}}+\frac{1}{\Omega_{c}^{2}}$. We consider three representative examples. (1) A frequencyanticorrelated state $\Phi_{1}$ [Fig. 1(d)] corresponds to $\theta=-\frac{\pi}{4}$. Such a state is commonly produced by type-I degenerate SPDC from a thin NLC [11]. The first term in Eq. (3) corresponds to the optical pump spectrum (bandwidth $\Omega_{\mathrm{c}}$ ) and the second term to phase matching ( $\Omega_{\mathrm{o}}$ is the phase-matching bandwidth). (2) A frequency-correlated state $\Phi_{2}$ [Fig. 1(e)] corresponds to $\theta=\frac{\pi}{4}$, produced using extended phase-matching schemes [6] or side-pumped single-mode nonlinear wave guides [9]. A strongly entangled state (for $\Phi_{1}$ or $\Phi_{2}$ ) results when $\Omega_{\mathrm{o}} \gg \Omega_{\mathrm{c}}$. (3) A separable 2P state $\Phi_{3}$ [Fig. 1(f)] corresponds to $\theta=0$ or $\frac{\pi}{2}$ [8], and a symmetric state is achieved when $\Omega_{\mathrm{o}} \ll \Omega_{\mathrm{c}}$. The coincidence rate produced by the general state in Eq. (3) is $G_{12}^{(2)}(\tau)=1-V \exp \left\{-\frac{\tau^{2}}{2 \tau_{0}^{2}}\right\}$, where the visibility $V$ and the width of the "dip" $\tau_{\mathrm{o}}$ are

$$
V=\sqrt{\frac{\left(1+\xi^{2}\right)^{2}-1}{\left(1+\xi^{2}\right)^{2}-\sin ^{2} 2 \theta}}, \quad \tau_{\mathrm{o}}^{2} \Omega_{\mathrm{o}}^{2}=1+\frac{1+\sin 2 \theta}{\xi^{2}},
$$

respectively, and $\xi=\sqrt{2} \Omega_{\mathrm{c}} / \Omega_{\mathrm{o}}$. We plot in Fig. $1(\mathrm{~g})-1(\mathrm{i})$ $G_{12}^{(2)}(\tau)$ that correspond to the states in Fig. 1(d)-1(f). In all three cases $V=1$. The width of $G_{12}^{(2)}(\tau)$ for $\Phi_{1}$ is $\tau_{\mathrm{o}}=\frac{1}{\Omega_{\mathrm{o}}}$; for $\Phi_{2}, \tau_{\mathrm{o}}^{2} \Omega_{\mathrm{o}}^{2}=1+\frac{2}{\xi^{2}}$, and $\tau_{\mathrm{o}} \rightarrow \frac{1}{\Omega_{\mathrm{c}}}$ when $\xi \rightarrow 0$, which results in a much broader dip than in the anticorrelated case when $\xi \ll 1[6]$; and the width of $G_{12}^{(2)}(\tau)$ for the separable state $\Phi_{3}$ (when $\xi \gg 1$ ) is similar to that of the entangled state $\Phi_{1}$ since they have the same reduced one-photon spectrum.

These examples highlight the ambiguities in state identification that inevitably arise in the HOM interferogram since it is a $1 \mathrm{D}$ temporal slice through the $2 \mathrm{P}$ state, which is $2 \mathrm{D}$ by definition. Placing two delays $\tau_{1}$ and $\tau_{2}$ in the two arms of the HOM-I [Fig. 1(a)] of course does not solve the problem. The new interferogram is $G^{(2)}\left(\tau_{1}, \tau_{2}\right)=G_{12}^{(2)}\left(\tau_{1}-\tau_{2}\right)$, i.e., the HOM interferogram is recovered but is parameterized instead by the relative delay $\tau_{1}-\tau_{2}$. The reason for this is clear since each photon takes one of the two paths to the detectors [Fig. 1(b)-1(c)], and placing a delay in a path thus affects both interfering $2 \mathrm{P}$ probability amplitudes whose phases are thus locked in opposition to each other.

We now describe a $2 \mathrm{P}$ interferometer based on the HOM-I that incorporates two independent delays $\tau_{1}$ and $\tau_{2}$, which relate separately to the frequencies $\omega_{1}$ and $\omega_{2}$ of each photon. To produce such a dual-delay $2 \mathrm{P}$ interferogram we need to unlock the fixed phase relationship between the interfering $2 \mathrm{P}$ probability amplitudes in Fig. 1(b)-1(c). The interferometer shown in Fig. 2(a) enables this unlocking by making a "copy"
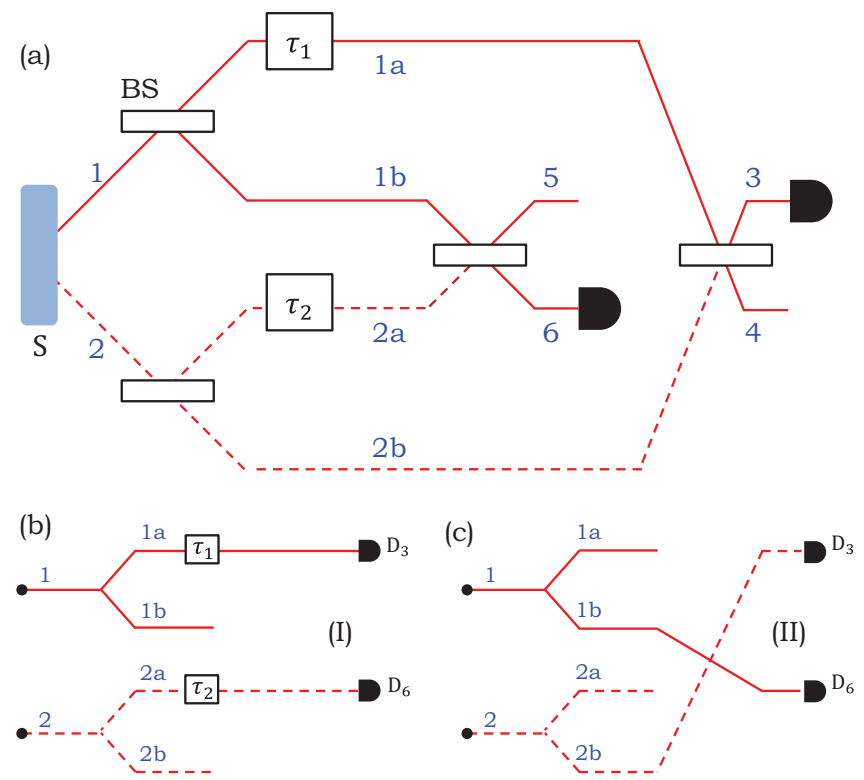

FIG. 2. (Color online) (a) Schematic of a dual-delay PhuL HOMI. S: $2 \mathrm{P}$ source, BS: beam splitter, $\tau_{1}, \tau_{2}$ : optical delays. (b)-(c) The two interfering $2 \mathrm{P}$ probability amplitudes (I) and (II), respectively (see text for details), that lead to a coincidence count, $G_{36}^{(2)}\left(\tau_{1}, \tau_{2}\right)$. The continuous and dashed lines identify photons 1 and 2 , respectively. 


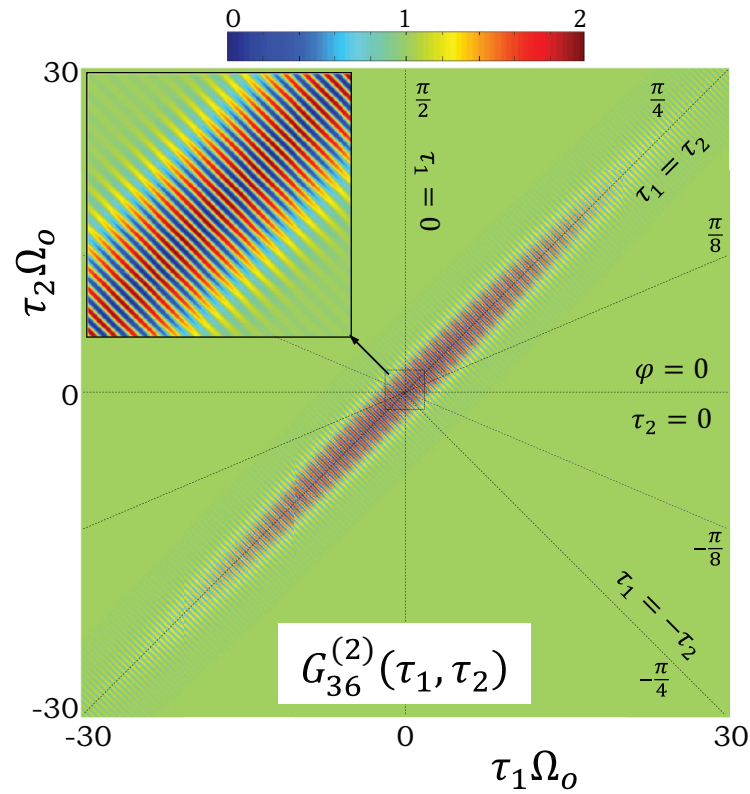

FIG. 3. (Color online) PhuL HOM interferogram $G_{36}^{(2)}\left(\tau_{1}, \tau_{2}\right)$ corresponding to $\Phi_{1}$ in Fig. 1(d). Each fringe along the $\tau_{1}+\tau_{2}=\tau_{\mathrm{d}}$ direction corresponds to a HOM interferogram. The ranges of $\tau_{1} \Omega_{0}$ and $\tau_{2} \Omega_{\mathrm{o}}$ in the inset are $[-4,4]$ and $\frac{\omega_{0}}{\Omega_{0}}=10$.

of each photon after splitting their paths with a BS: paths 1a and $1 b$ for photon 1 , and $2 a$ and $2 b$ for photon 2 . We place delay $\tau_{1}$ in path 1a of photon 1 and a second delay $\tau_{2}$ in path $2 \mathrm{a}$ of photon 2. Paths $1 \mathrm{a}$ and $2 \mathrm{~b}$ ( $1 \mathrm{~b}$ and $2 \mathrm{a}$ ) are brought together at a BS with output ports 3 and 4 (5 and 6).

A new interferogram is revealed in $G_{36}^{(2)}\left(\tau_{1}, \tau_{2}\right)$. Two $2 \mathrm{P}$ probability amplitudes lead to a coincidence count at $\mathrm{D}_{3}$ and $\mathrm{D}_{6}$ : (I) photon 1 in path $1 \mathrm{a}$ (delay $\tau_{1}$ ) reaches $\mathrm{D}_{3}$ while photon 2 in path $2 \mathrm{a}$ (delay $\tau_{2}$ ) reaches $\mathrm{D}_{6}$ [Fig. 2(b)]; or (II) photon 1 in path $1 \mathrm{~b}$ reaches $\mathrm{D}_{6}$ while photon 2 in path $2 \mathrm{~b}$ reaches $\mathrm{D}_{3}$ [Fig. 2(c)]. The $2 \mathrm{P}$ probability amplitude (II) is delay-free and serves as a $2 \mathrm{P}$ reference against which the $2 \mathrm{P}$ probability amplitude (I) beats. The interfering $2 \mathrm{P}$ probability amplitudes in the PhuL HOM-I are thus no longer locked in antiphase as is the case for the corresponding $2 \mathrm{P}$ probability amplitudes in the HOM-I. Note that the interfering $2 \mathrm{P}$ probability amplitudes do not include the two photons meeting at the BSs nor overlapping in any path in the interferometer. Nevertheless, HOM interference takes place since such an effect relies only on the indistinguishability of the interfering $2 \mathrm{P}$ probability amplitudes.

Starting with the general $2 \mathrm{P}$ state in Eq. (1), the time-averaged dual-delay normalized coincidence rate is $G_{36}^{(2)}\left(\tau_{1}, \tau_{2}\right)=1-\Re\left\{\Gamma\left(\tau_{1}, \tau_{2}\right)\right\}$, where

$$
\Gamma\left(\tau_{1}, \tau_{2}\right)=\iint d \omega_{1} d \omega_{2} \Phi\left(\omega_{1}, \omega_{2}\right) \Phi^{*}\left(\omega_{2}, \omega_{1}\right) e^{-i\left\{\omega_{1} \tau_{1}+\omega_{2} \tau_{2}\right\}},
$$

which is the $2 \mathrm{D}$ Fourier transform of the symmetrized state function. The two delays $\tau_{1}$ and $\tau_{2}$ relate independently to the two frequencies $\omega_{1}$ and $\omega_{2}$. Taking the coincidence rate at $\mathrm{D}_{3}$ and $\mathrm{D}_{5}$ instead, $G_{35}^{(2)}\left(\tau_{1}, \tau_{2}\right)$ is identical to $G_{36}^{(2)}\left(\tau_{1}, \tau_{2}\right)$ except for a change in the sign of the interference term.

There are several general statements that can be made about this interferogram before discussing specific examples. First, comparing Eq. (2) with Eq. (5) reveals that the HOM interference term is the antidiagonal through $\Gamma\left(\tau_{1}, \tau_{2}\right)$ : $\Lambda(\tau)=\Gamma(\tau,-\tau)$. In other words, by sweeping $\tau_{1}$ and $\tau_{2}$ in opposition $\left(\tau_{1}=-\tau_{2}\right)$, then $G_{36}^{(2)}(\tau,-\tau)=1-\Lambda(\tau)$ reproduces the HOM interferogram, which is related to the uncertainty in the difference between the arrival times of the two photons. Second, sweeping $\tau_{1}$ and $\tau_{2}$ in unison $\left(\tau_{1}=\tau_{2}\right)$ reveals an "orthogonal" $2 \mathrm{P}$ interferogram $G_{36}^{(2)}(\tau, \tau)$ that has not been observed experimentally heretofore, and that is related to the absolute arrival times of the photon pairs, as we show below. Third, since $\Gamma\left(\tau_{1}, \tau_{2}\right)=\Gamma^{*}\left(-\tau_{2},-\tau_{1}\right)$, then $\Gamma(\tau,-\tau)$ is real while $\Gamma(\tau, \tau)$ is not necessarily so, and $\Re\left\{\Gamma\left(\tau_{1}, \tau_{2}\right)\right\}$ has mirror symmetry around $\tau_{1}+\tau_{2}=0$. Finally, note that the $1 \mathrm{D}$ interferograms $G_{36}^{(2)}(\tau, 0)$ and $G_{36}^{(2)}(0, \tau)$ that result from fixing one of the two delays do not correspond to the HOM interferogram. The corresponding interference terms are $\Gamma(\tau, 0)=\int d \omega \Phi_{1}(\omega) e^{-i \omega \tau}$ and $\Gamma(0, \tau)=\Gamma^{*}(-\tau, 0)$, respectively, where $\Phi_{1}(\omega)=\int d \omega^{\prime} \Phi\left(\omega, \omega^{\prime}\right) \Phi^{*}\left(\omega^{\prime}, \omega\right)$.

Sweeping $\tau_{1}$ and $\tau_{2}$ independently produces a $2 \mathrm{D}$ PhuL HOM interferogram that lifts the ambiguities highlighted in Fig. 1. Using the model state in Eq. (3), we obtain $G_{36}^{(2)}\left(\tau_{1}, \tau_{2}\right)=$

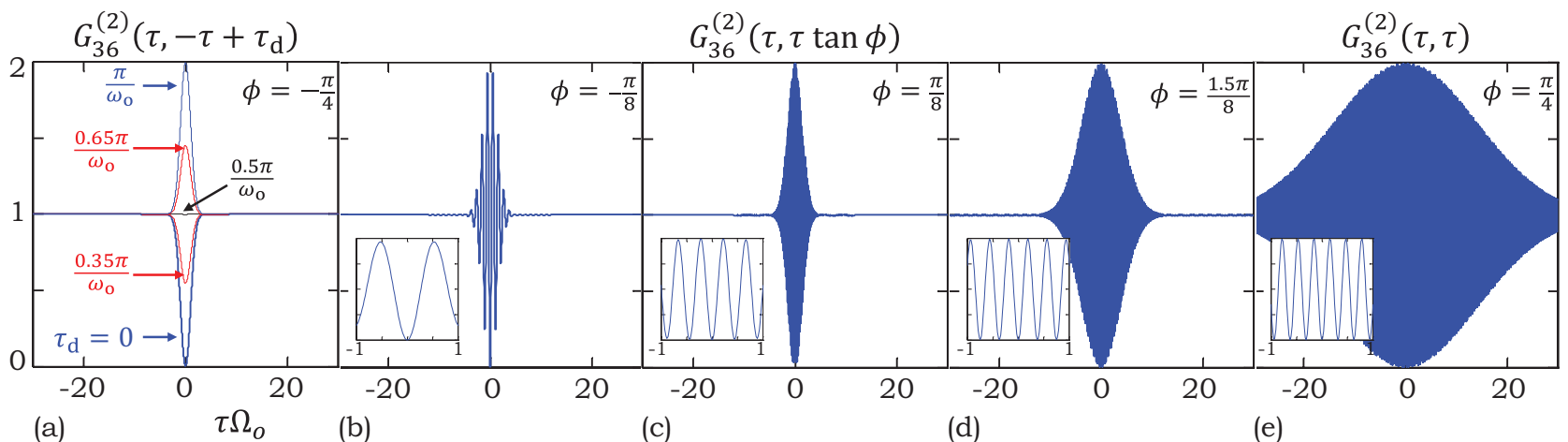

FIG. 4. (Color online) HOM interferograms obtained from $G_{36}^{(2)}\left(\tau_{1}, \tau_{2}\right)$ (Fig. 3) by fixing a linear relation between $\tau_{1}$ and $\tau_{2}$. (a) $G_{36}^{(2)}(\tau$, $\tau+\tau_{\mathrm{d}}$ ) along $\phi=-\frac{\pi}{4}$ in Fig. 3. When $\tau_{\mathrm{d}}=0$ we obtain the usual HOM interferogram. Changing $\tau_{\mathrm{d}}$ from 0 to $\frac{\pi}{\omega_{\mathrm{o}}}$, the HOM "dip"” becomes a "peak." (b)-(e) $\tau_{2}=\tau_{1} \tan \phi$ : (b) $\phi=-\frac{\pi}{8}$, (c) $\phi=\frac{\pi}{8}$, (d) $\phi=\frac{1.5 \pi}{8}$, and (e) $\phi=\frac{\pi}{4}$, corresponding to $G_{36}^{(2)}(\tau, \tau)$. Insets show the range $[-1,1]$ of $\tau \Omega_{\mathrm{o}}$ to identify the modulating angular frequency, $\omega_{o}(1+\tan \phi)$; here $\frac{\omega_{0}}{\Omega_{0}}=10$. 

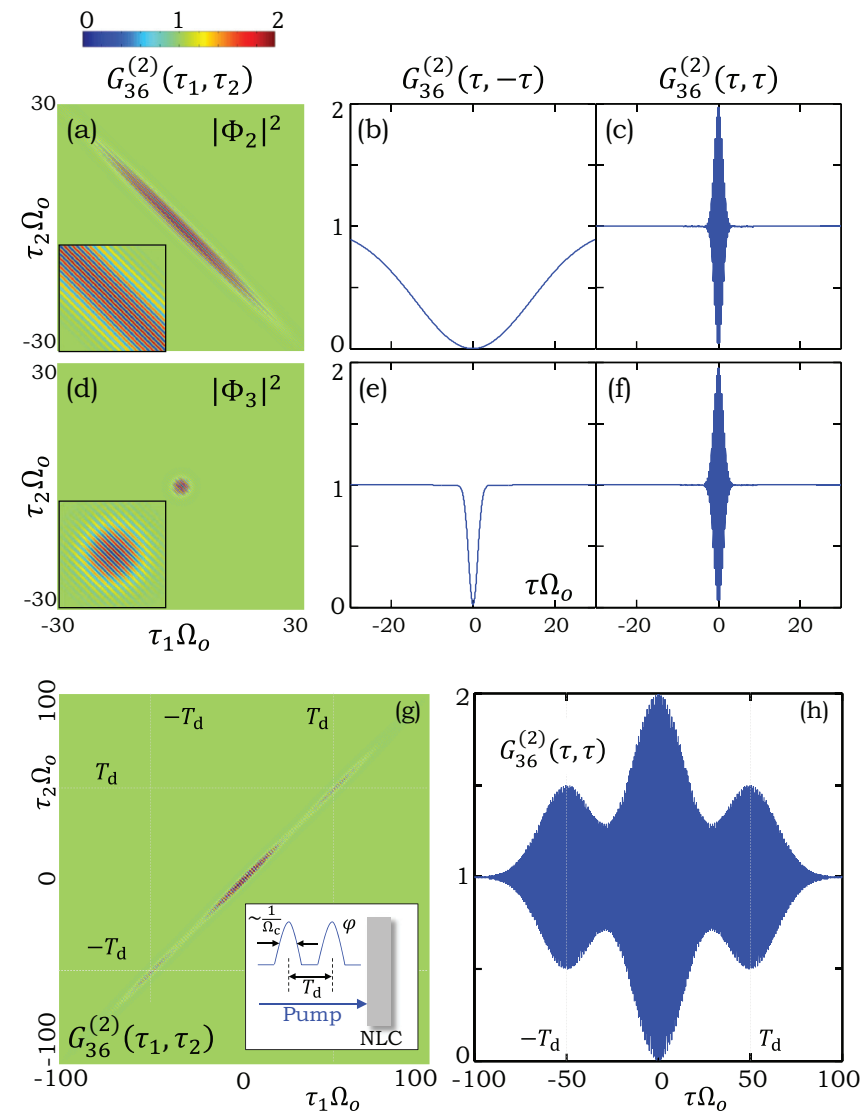

FIG. 5. (Color online) (a)-(c) 2P PhuL HOM interferograms $G_{36}^{(2)}\left(\tau_{1}, \tau_{2}\right), G_{36}^{(2)}(\tau,-\tau)$, and $G_{36}^{(2)}(\tau, \tau)$ for the frequency-correlated state $\Phi_{2}$ [Fig. 1(e)]. (d)-(f) Same as in (a)-(c) for the separable 2P state $\Phi_{3}$ [Fig. 1(f)]. The ranges of $\tau_{1} \Omega_{\mathrm{o}}$ and $\tau_{2} \Omega_{\mathrm{o}}$ in the insets in (a) and (d) are all $[-4,4]$ and $\frac{\omega_{0}}{\Omega_{0}}=10 .(\mathrm{g}) G_{36}^{(2)}\left(\tau_{1}, \tau_{2}\right)$ and (h) $G_{36}^{(2)}(\tau, \tau)$ for frequency-anticorrelated type-I SPDC from a NLC pumped by a pulse doublet. Here $\xi=0.1$, the interpulse delay is $T_{\mathrm{d}} \Omega_{\mathrm{o}}=50$, $\varphi=0$, and $\frac{\omega_{0}}{\Omega_{0}}=10$.

$1-V \cos \left\{\omega_{\mathrm{o}}\left(\tau_{1}+\tau_{2}\right)\right\} \Gamma_{\mathrm{o}}\left(\tau_{1}, \tau_{2}\right)$, where

$$
\Gamma_{\mathrm{o}}\left(\tau_{1}, \tau_{2}\right)=\exp \left\{-\frac{\tau_{2}^{2}}{2 T_{\mathrm{o}}^{2}}\right\} \exp \left\{-\frac{\left(\tau_{1}+\delta \tau_{2}\right)^{2}}{2 T_{\mathrm{o}}^{2}\left(1-\delta^{2}\right)}\right\} ;
$$

here $T_{\mathrm{o}}^{2} \Omega_{\mathrm{o}}^{2}=2\left(1+\frac{1}{\xi^{2}}\right), \delta=\frac{\sin 2 \theta}{1+\xi^{2}}$, and $V$ is given in Eq. (4). When $\tau_{1}=-\tau_{2}$, this $2 \mathrm{D}$ interferogram reduces to the $1 \mathrm{D} \mathrm{HOM}$ interferogram with $\tau_{\mathrm{o}}=T_{\mathrm{o}} \sqrt{1+\delta}$.

We first consider the frequency-anticorrelated state $\Phi_{1}$ whose 2D PhuL HOM interferogram is shown in Fig. 3. The general features of the PhuL HOM interferogram discussed above are easily recognized here. On the $\tau_{1}+\tau_{2}=0$ axis we retrieve the HOM interferogram [Fig. 4(a)]. Furthermore, when the two delays have an offset $\tau_{\mathrm{d}}$, the interferograms along $\tau_{1}+\tau_{2}=\tau_{\mathrm{d}}$ cycle periodically between a "dip" (signifying that the two photons never reach detectors $D_{3}$ and $D_{6}$ simultaneously) and a "peak" (signifying that they always reach these detectors together), since the interference term is weighed by $\cos \omega_{\mathrm{o}} \tau_{\mathrm{d}}$ [Fig. 4(a)]. If the delays are swept such that $\tau_{2}=\tau_{1} \tan \phi, 1 \mathrm{D}$ sections with slope $\tan \phi$ through the $2 \mathrm{D}$ PhuL HOM interferogram are obtained along lines through the origin. As $\phi$ increases from $-\frac{\pi}{4}$ to $\frac{\pi}{4}$, (1) the width evolves from $\frac{1}{\Omega_{\mathrm{o}}}$ to $\frac{1}{\Omega_{\mathrm{c}}}$ and (2) a sinusoid modulates the interferogram with frequency $(1+\tan \phi) \omega_{\mathrm{o}}$ ranging from 0 at $\phi=-\frac{\pi}{4}$ (the HOM "dip") to $\omega_{\mathrm{o}}$ at $\phi=0$ and further to $2 \omega_{\mathrm{o}}$ at $\phi=\frac{\pi}{4}$.

The 2D PhuL-HOM interferograms for the frequencycorrelated $\left(\Phi_{2}\right)$ and separable $\left(\Phi_{3}\right) 2 \mathrm{P}$ states are shown in Fig. 5 along with the $1 \mathrm{D}$ antidiagonal $\left(\tau_{1}=-\tau_{2}\right)$ and diagonal $\left(\tau_{1}=\tau_{2}\right)$ interferograms. We see clearly that the role of the 1D interferograms for the frequency-correlated state are reversed with respect to the frequency-anticorrelated state [compare Fig. 4(a) with Fig. 5(b) and Fig. 4(e) with Fig. 5(c)]. The separable state is characterized by a separable 2D PhuL HOM interferogram [Fig. 5(d)] having equal widths of the 1D interferograms along any direction through the origin [Figs. 5(e)-5(f)].

To further highlight the usefulness of the PhuL HOM-I, we examine the $2 \mathrm{P}$ state produced by a NLC pumped with a pulse doublet consisting of identical pairs of pulses of width $\frac{2 \pi}{\Omega_{\mathrm{c}}}$ separated by a delay $T_{\mathrm{d}}$ larger than the pulse width but shorter than the detector time window, in addition to having a relative phase $\varphi$ [Fig. 5(g), inset]. The HOM interferogram $G_{12}^{(2)}(\tau)$ produced by this state for all values of $T_{\mathrm{d}}$ and $\varphi$ is identical to that produced by the $2 \mathrm{P}$ state when a single-pulse pump is used. The distinction between these two cases is easily made in the PhuL HOM-I [21] by examining the $\tau_{1}=\tau_{2}$ axis, which is inaccessible to the HOM-I, where we find two new features at $G_{36}^{(2)}(\tau, \tau)$ and $\tau= \pm T_{\mathrm{d}}$ [Fig. 5(g)]. Moreover, $G_{36}^{(2)}\left( \pm T_{\mathrm{d}}, \pm\right.$ $\left.T_{\mathrm{d}}\right)=1-\frac{1}{2} \cos \left(2 \omega_{\mathrm{o}} T_{\mathrm{d}}-\varphi\right)$ is controlled through modulating the value of $\varphi$, while the traditional HOM interferogram (along $\tau_{1}+\tau_{2}=0$ ) remains invariant. Clearly, the $\tau_{1}-\tau_{2}=0$ axis corresponds to the absolute arrival times of the photon pairs, revealed in the autocorrelation of the pulse doublet in Fig. 5(h).

Finally, the HOM-I and PhuL HOM-I may be extended to other degrees of freedom, such as orbital angular momentum, by replacing the delays with so-called general phase operators [22], thereby enabling the analysis of $2 \mathrm{P}$ states in an arbitrary basis [23].

In conclusion, we have introduced a linear $2 \mathrm{P}$ interferometer comprising two delays capable of producing a 2D interferogram by spanning independently the two temporal dimensions of 2P states. Because the PhuL HOM-I allows access to an "orthogonal" temporal dimension hidden from the HOM-I, 2P states that have similar HOM interferograms or ones that yield featureless HOM interferograms become identifiable, thus making the PhuL HOM-I useful in characterizing engineered $2 \mathrm{P}$ states and synchronization of two photons in an arbitrary state.
[1] C. K. Hong, Z. Y. Ou, and L. Mandel, Phys. Rev. Lett. 59, 2044 (1987).

[2] G. Di Giuseppe, M. Atatüre, M. D. Shaw, A. V. Sergienko, B. E. A. Saleh, M. C. Teich, A. J. Miller, S. W. Nam, and J. Martinis, Phys. Rev. A 68, 063817 (2003).
[3] A. Zeilinger, Phys. Scr. 1998, 203 (1998); Z. Y. J. Ou, Multi-Photon Quantum Interference (Springer, New York, 2007).

[4] J.-W. Pan et al., Nature (London) 403, 515 (2000). 
[5] J.-W. Pan, D. Bouwmeester, H. Weinfurter, and A. Zeilinger, Phys. Rev. Lett. 80, 3891 (1998); J.-W. Pan et al., Rev. Mod. Phys. 84, 777 (2012).

[6] V. Giovannetti, L. Maccone, J. H. Shapiro, and F. N. C. Wong, Phys. Rev. Lett. 88, 183602 (2002); Phys. Rev. A 66, 043813 (2002); O. Kuzucu, M. Fiorentino, M. A. Albota, F. N. C. Wong, and F. X. Kärtner, Phys. Rev. Lett. 94, 083601 (2005).

[7] A. B. U'Ren, C. Silberhorn, K. Banaszek, and I. A. Walmsley, Phys. Rev. Lett. 93, 093601 (2004).

[8] P. J. Mosley et al., New J. Phys. 10, 093011 (2008).

[9] Z. D. Walton, M. C. Booth, A. V. Sergienko, B. E. A. Saleh, and M. C. Teich, Phys. Rev. A 67, 053810 (2003); 70, 052317 (2004).

[10] J. P. Torres et al., Opt. Lett. 30, 314 (2005); A. Valencia, A. Ceré, X. Shi, G. Molina-Terriza, and J. P. Torres, Phys. Rev. Lett. 99, 243601 (2007).

[11] S. E. Harris, M. K. Oshman, and R. L. Byer, Phys. Rev. Lett. 18, 732 (1967); B. E. A. Saleh, A. F. Abouraddy, A. V. Sergienko, and M. C. Teich, Phys. Rev. A 62, 043816 (2000).

[12] S. Sensarn, G. Y. Yin, and S. E. Harris, Phys. Rev. Lett. 104, 253602 (2010).

[13] O. Kuzucu F. N. C. Wong, S. Kurimura, and S. Tovstonog, Phys. Rev. Lett. 101, 153602 (2008).

[14] Y.-H. Kim and W. P. Grice, Opt. Lett. 30, 908 (2005); M. Hendrych, M. Mičuda, and J. P. Torres, ibid. 32, 2339 (2007).

[15] W. Wasilewski, P. Wasylczyk, P. Kolenderski, K. Banaszek, and C. Radzewicz, Opt. Lett. 31, 1130 (2006).
[16] In Ref. [15] the two photons are directed to uncoupled onephoton interferometer and a 2D 2P interferogram is obtained. Post-selection then filters the desired 2P interference terms while eliminating one-photon terms. This configuration is useful for narrow-band spectra where the various terms do not overlap. This configuration cannot be used in the other applications of the HOM-I, such as photon synchronization used in entanglement swapping.

[17] V. Giovannetti, S. Lloyd, and L. Maccone, Nature (London) 412, 417 (2001).

[18] A. F. Abouraddy M. B. Nasr, B. E. A. Saleh, A. V. Sergienko, and M. C. Teich, Phys. Rev. A 65, 053817 (2002).

[19] T. B. Pittman, D. V. Strekalov, A. Migdall, M. H. Rubin, A. V. Sergienko, and Y. H. Shih, Phys. Rev. Lett. 77, 1917 (1996).

[20] R. J. Glauber, Phys. Rev. 130, 2529 (1963).

[21] The PhuL HOM interferogram here is given by

$$
\begin{aligned}
G_{36}^{(2)}\left(\tau_{1}, \tau_{2}\right)= & 1-\cos \left\{\omega_{\mathrm{o}}\left(\tau_{1}+\tau_{2}\right)\right\} \Gamma_{\mathrm{o}}\left(\tau_{1}, \tau_{2}\right) \\
& -\frac{1}{2} \cos \left\{\omega_{\mathrm{o}}\left(\tau_{1}+\tau_{2}\right)-\varphi\right\} \Gamma_{\mathrm{o}}\left(\tau_{1}-T_{\mathrm{d}}, \tau_{2}-T_{\mathrm{d}}\right) \\
& -\frac{1}{2} \cos \left\{\omega_{\mathrm{o}}\left(\tau_{1}+\tau_{2}\right)+\varphi\right\} \Gamma_{\mathrm{o}}\left(\tau_{1}+T_{\mathrm{d}}, \tau_{2}+T_{\mathrm{d}}\right),
\end{aligned}
$$

where $\Gamma_{\mathrm{o}}\left(\tau_{1}, \tau_{2}\right)$ is given in Eq. (6).

[22] A. F. Abouraddy, T. M. Yarnall, and B. E. A. Saleh, Opt. Lett. 36, 4683 (2011); 37, 2889 (2012).

[23] T. M. Yarnall, G. Di Giuseppe, and A. F. Abouraddy (unpublished). 